\section{Submitted from BMJ Quality}

\title{
Improving the management of iron deficiency in ambulatory heart failure patients
}

\author{
Carl Hayward, Hitesh Patel, Chris Allen, Ali Vazir
}

To cite: Hayward C, Patel $\mathrm{H}$, Allen $\mathrm{C}$, et al. Improving the management of iron deficiency in ambulatory heart failure patients. BMJ Quality Improvement Reports 2016;5:u209822.w4076. doi:10.1136/bmjquality. u209822.w4076

Received 10 March 2016 Revised 26 April 2016

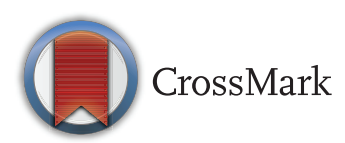

Royal Brompton Hospital, UK

Correspondence to Carl Hayward c.hayward@ rbht.nhs.uk2016

\section{ABSTRACT}

Based on clinical trial data patients with heart failure (HF) and evidence of iron deficiency should be offered intravenous (iv) iron with the aim of improving exercise capacity and symptoms. Baseline measurement in outpatient HF clinics demonstrated that only $50 \%$ of patients who may be eligible for iv iron were investigated with iron studies. Our aim was to make sure that $90 \%$ of the patients attending our heart failure clinics who were symptomatic and had an ejection fraction (EF) $\leq 45 \%$ should have their iron studies checked within the last six months.

In an effort to increase the proportion of suitable patients in whom iron studies are requested, we carried out three plan-do-study-act (PDSA) cycles each with a different intervention. These interventions included a presentation of the clinical trial evidence at a HF multidisciplinary meeting, email reminders prior to clinic and stickers in the patient notes (repeated twice). The effect of each intervention was measured with the outcome being the proportion of eligible patients in whom iron studies were documented within the previous 6 months. The interventions increased the number of suitable patients who had iron studies checked, to as high as $100 \%$, however this effect was not sustained. Root cause analysis revealed that clinicians were unenthusiastic to continue performing iron studies due to inefficiency in the process of admitting patients and giving them iv iron. For example median in-hospital stay of seven hours for an infusion that is given over 15 minutes.

In an attempt to improve patient and physician satisfaction we piloted an ambulatory outpatient service to deliver iv iron. We demonstrated that this service was feasible and more efficient as less time was required waiting for a bed or spent in hospital and was less costly. In summary we have demonstrated interventions which can increase the identification of patients who would benefit from iv iron and piloted a new time and cost efficient system of administration of iv iron.

\section{PROBLEM}

Patients with heart failure who are iron deficient achieve symptomatic benefit from iv iron therapy. Iron deficiency can be diagnosed by an iron studies blood test. Despite this, the diagnosis of iron deficiency in patients with heart failure is not widely sought by the heart failure community. This is an area in need of quality improvement. ${ }^{1}$

The Royal Brompton Hospital, London is a tertiary referral centre for cardiovascular diseases in England. Over 1000 heart failure patients/year are seen in our heart failure outpatient clinics (four consultant clinics/week).

The goal of this quality improvement project is to ensure that, $90 \%$ of patients attending our heart failure clinics that are symptomatic and have an ejection fraction (EF) $\leq 45 \%$ should have had their iron studies checked within the last six months. $90 \%$ was chosen as this is approximately the proportion of our heart failure patients in whom we achieve maintenance therapy on prognosis altering drugs such as beta-blockers.

Our team consisted of heart failure doctors (consultants, registrar, and senior house officers), nurse specialists, and cardiac pharmacists.

\section{BACKGROUND}

Iron deficiency is present in approximately $30-50 \%$ of patients with chronic heart failure in the ambulatory setting. ${ }^{2}$ Patients with heart failure compounded with iron deficiency have worse symptoms and cardiovascular outcomes than those that do not. ${ }^{3}$ Randomised placebo-controlled trials have suggested that replenishing iron stores using an intravenous infusion can improve patient symptoms and reduce their need for heart failure related hospital admissions., ${ }^{4,5}$ The patients in these trials were symptomatic (New York Heart Association class: 2-3) and had an EF $<45 \%$. The European Society of Cardiology recommends that all heart failure patients should be assessed for iron deficiency using the following defined cut-offs: ferritin $<100 \mathrm{ng} / \mathrm{L}$ or ferritin $<300 \mathrm{ng} / \mathrm{L}$ with transferrin saturation $<20 \%{ }^{6}$

Parenteral iron is more effective than oral supplementation in replenishing iron stores 
in heart failure. ${ }^{7}$ The former is also better tolerated. The most significant side-effect of intravenous iron is hypersensitivity/anaphylaxis, which is rare with an incidence of $<1 / 1000$ and in post-marketing data no fatalities have been reported. Consequently, many centres are now administering parenteral iron in an outpatient setting as a 15 minute infusion. Once administered another dose may not be required for several months dependent on whether the patient remains iron deficient.

\section{BASELINE MEASUREMENT}

We analysed the records of all patients who attended our heart failure clinics in February 2015. In this month, 40 patients had symptomatic heart failure with an $\mathrm{EF} \leq 45 \%$. Of these, only $20(50 \%)$ had iron levels checked within the previous six months. All of these patients who had iron levels checked were found to be iron deficient using the recommended cut-off values for ferritin and transferrin saturations. None of these patients received intravenous iron. These data suggest that we are not following international or local guidelines on assessing all symptomatic heart failure patients for iron deficiency.

\section{DESIGN}

When we presented our baseline data at the heart failure multidisciplinary meeting the opinion of the heart failure team was that this is an important area and that we should be more proactive in identifying and treating iron deficiency. To establish a measurable outcome it was agreed by consensus that patients who met the clinical criteria should have iron studies performed at least once every six months.

Our baseline data suggest two areas for potential improvement: 1) A large proportion of symptomatic heart failure patients were not being investigated for iron deficiency (Figure 1) and 2) Those found to be iron deficient were not being offered appropriate management advice.

Our approach to improve these findings was to increase awareness and education regarding the importance of diagnosing and methods of treating iron deficiency in heart failure.

This study was a quality improvement project and hence did not require ethical approval and it was formally registered at the Royal Brompton Hospital.

\section{STRATEGY}

Three Plan-Do-Study-Act (PDSA) cycles were performed. We assessed the effect of each intervention by determining the proportion of symptomatic patients with an EF $<45 \%$ that were tested for iron deficiency in the six months before clinic or on the day of clinic. The process outcome was measured weekly for all patients attending a heart failure clinic that week. Each intervention was communicated formally (presentation at meetings, email, letter) and informally (corridor conversations) to the whole heart failure team.

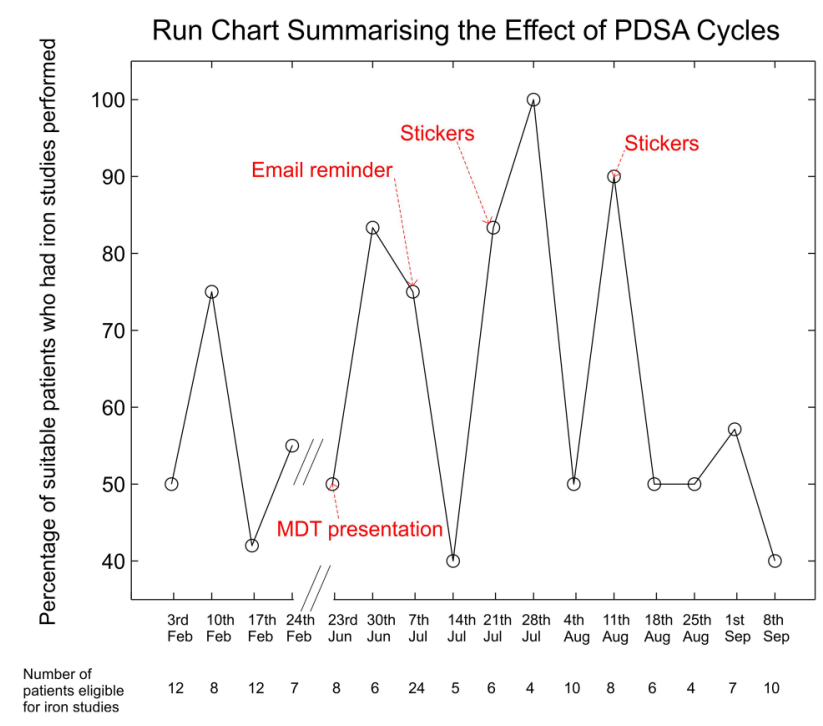

Figure 1

PDSA cycle 1: Our initial approach was to present the baseline measurements to the heart failure team at the weekly multi-disciplinary meeting, which is attended by consultants, junior doctors, specialist nurses, and pharmacists. We also discussed the European Society of Cardiology guidelines and key randomised controlled trials. In this group setting it was agreed that our symptomatic heart failure patients ought to be tested for iron deficiency at least every six months and if found to be deficient treatment options discussed.

PDSA cycle 2: An email reminder was sent to the heart failure team the day before clinic reiterating the conclusions of the multi-disciplinary team meeting and highlighting the importance of diagnosing iron deficiency.

PDSA cycle 3: A sticker (see Figure 2) was inserted on the cover sheet of each set of clinic notes as a further measure to remind the clinical team to consider investigating for iron deficiency in appropriate patients. This intervention was repeated after a fortnight.

\section{RESULTS}

The effect of each PDSA cycle on the proportion of suitable patients in whom iron studies were performed is demonstrated in the run chart (Figure 2). Using $50 \%$ as

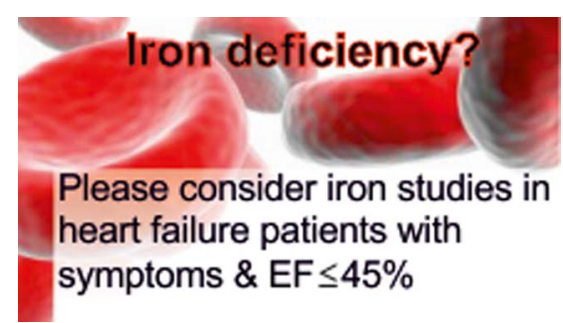

Figure 2 Sticker 
the median number of patients who were tested for iron deficiency at baseline, there were no shifts or trends and the number of runs $(n=4)$ did not support a signal of change. After some of our interventions we were able to increase the proportion of patients tested from $50 \%$ to $100 \%$, however, this effect waned with time. An explanation for this was that despite more iron deficient patients being identified, no patients actually received intravenous iron during our study time period. Through root cause analysis we identified several factors that may explain why iron deficient patients are not receiving iron therapy in our cohort: 1) Our patients are not local to our hospital and hence it may be inconvenient for them to come back to our services to receive iv iron; 2) Currently, iv iron administration in our trust requires an admission to a hospital bed, which is a limited resource and hence patients are likely to join a long waiting list; 3) When patients come in to receive the iron infusion, which actually takes 15-30mins, their actual 'admission' lasts a median of seven hours as delays are introduced at several stages including waiting for: an empty bed, cannulation, prescription and dispensation of the iron preparation, administration of the preparation by the nurse, and finally discharge paper work; and 4) In the current set-up intravenous iron administration leads to a financial loss of $£ 157.58$ per admission for the trust.

After completion of the PDSA cycles and the root cause analysis, we piloted a new service to improve the patient journey for those receiving iv iron and to improve satisfaction amongst physicians referring patients for this therapy. Firstly, we developed a patient information leaflet to explain the implications of iron deficiency in heart failure, the potential treatment options and what to expect if they are offered iv iron. We then performed a feasibility assessment of administration of intravenous iron in the outpatient setting on one patient. This case study resulted in a hospital stay of only one hour and 15 minutes with the added benefit of improved subjective patient satisfaction (the previous hospital admission model had led to several complaints related to the waiting times) and a financial gain to the trust of $£ 332.78$ (as opposed to a loss of $£ 157.58$ in the current system).

\section{LESSONS AND LIMITATIONS}

We have learnt numerous lessons from this quality improvement project: 1) It is important to start and is often easier to do so by introducing a small change with the goal of making a small improvement rather than spending an excessive amount of time trying to achieve perfection from the outset. 2) Our outcome was ultimately shown to be unsustainable as the final process (iv iron infusion) was in its current state both resource inefficient and patient unfriendly, deterring clinicians from recommending it. This issue might have been highlighted at an earlier stage in the quality improvement process had we chosen to undertake a thorough process map with the stakeholders. 3) It is valuable to continuously evaluate the findings, in particular if results are not as expected a root-cause analysis might help redirect the focus of the project. In our case it directed our attention to the unpalatable set-up of the iv iron delivery process.

We hope that the lessons we have learned from our work might be adaptable by those seeking to embark on similar quality improvement projects.

\section{CONCLUSION}

Raising the awareness with recurrent reminders of the importance of iron deficiency in heart failure improved the proportion of patients tested, however, this was not sustained and did not lead to an increase in the number of patients treated with intravenous iron. This may be due to a lack of confidence in our current system of delivering iv iron, which requires a hospital admission, which is neither cost-effective nor patient-centred. Others seeking to perform a similar project to ours would be well served by performing a process map with all the stakeholders (including potential patients) early as well as undertaking root cause analysis if the initial results do not show a sustained improvement.

We have also demonstrated the feasibility of delivering iv iron in an outpatient setting which saved both time and money. The next step in this quality improvement project will be to put a business case forward for setting up an out-patient intravenous iron service so that more patients can benefit from this therapy in our institute.

Acknowledgements We are grateful to Dr Andrew Morley-Smith for his contribution to data collection.

Declaration of interests We have read and understood BMJ policy on declaration of interests and declare that we have no competing interests.

Ethical approval Formal ethical approval was not required as this was a quality improvement project and this was registered with our hospital's Research and Audit office prior to commencement.

Open Access This is an open-access article distributed under the terms of the Creative Commons Attribution Non-commercial License, which permits use, distribution, and reproduction in any medium, provided the original work is properly cited, the use is non commercial and is otherwise in compliance with the license. See:

- http://creativecommons.org/licenses/by-nc/2.0/

- http://creativecommons.org/licenses/by-nc/2.0/legalcode

\section{REFERENCES}

1. Silverberg DS, Schwartz D, Schwartz I, Ben Assa E. The missed opportunities to diagnose and treat iron deficiency in patients hospitalized with heart failure. Int J Cardiol 2013;168:2164-66.

2. Klip IT, Comin-Colet J, Voors AA, et al. Iron deficiency in chronic heart failure: an international pooled analysis. Am Heart $J$ 2013;165:575-82 e573.

3. Comin-Colet J, Enjuanes C, Gonzalez G, et al. Iron deficiency is a key determinant of health-related quality of life in patients with chronic heart failure regardless of anaemia status. Eur J Heart Fail 2013;15:1164-72.

4. Ponikowski $\mathrm{P}$, van Veldhuisen DJ, Comin-Colet J, et al. Beneficial effects of long-term intravenous iron therapy with ferric carboxymaltose in patients with symptomatic heart failure and iron deficiencydagger. Eur Heart J 2015;36:657-68. 
5. Anker SD, Comin Colet J, Filippatos G, et al. Ferric carboxymaltose in patients with heart failure and iron deficiency. $N$ Engl $J$ Med 2009;361:2436-48.

6. McMurray JJ, Adamopoulos S, Anker SD, et al. ESC guidelines for the diagnosis and treatment of acute and chronic heart failure 2012: The Task Force for the Diagnosis and Treatment of Acute and
Chronic Heart Failure 2012 of the European Society of Cardiology. Developed in collaboration with the Heart Failure Association (HFA) of the ESC. Eur J Heart Fail 2012;14:803-69.

7. McDonagh T, Macdougall IC. Iron therapy for the treatment of iron deficiency in chronic heart failure: intravenous or oral? Eur $J$ Heart Fail 2015;17:248-62. 